\title{
Complete Genome Sequence of Stenotrophomonas rhizophila KC1, a Quorum Sensing-Producing Algicidal Bacterium Isolated from Mangrove Kandelia candel
}

\author{
Qin Zhang, ${ }^{1}$ Yan Wang, ${ }^{2, \dagger}$ and Jin Zhou ${ }^{3, \dagger}$ \\ ${ }^{1}$ University of Queensland, Brisbane, Australia \\ ${ }^{2}$ Shenzhen Polytechnic, Shenzhen 518055, P. R. China \\ ${ }^{3}$ Shenzhen International Graduate School, Tsinghua University, Shenzhen 518055, P. R. China
}

\begin{abstract}
This paper describes the isolation of an algicidal strain, Stenotrophomonas rhizophila $\mathrm{KC} 1$, from mangrove (Kandelia candel) and its genome, which was sequenced using next-generation sequencing technology. The genome is $5.93 \mathrm{Mb}$ with a $\mathrm{G}+\mathrm{C}$ content of $63.17 \%$. A total of 3,352 functional proteins were assigned according to Kyoto Encyclopedia of Genes and Genomes categories. A total of 11,586 protein-coding genes, 73 transfer RNA genes, and 17 ribosomal RNA genes were obtained. In silico genome annotation protocols identified 83 putative quorum sensing (QS) genes, and the algicidal potential of KC1 was related with the QS genes (for example LuxI-LuxR genes). Collectively, these data suggest that KC1 may be an antialgal bacterium whose behavior can be modulated by QS signaling. The annotated genome sequence of this strain may represent a valuable tool for studying algae-bacteria interactions and developing microbe-based methods for controlling harmful algae.
\end{abstract}

\section{Genome Announcement}

Harmful algal blooms (HABs) are caused by the rapid proliferation of algae in response to eutrophication and pose a significant threat to both the environment and human health (Anderson 2009; Medlin 2013). HABs can intoxicate humans via the ingestion of contaminated seafood, reduce stocks of wild or cultured fish, and impair tourism (Anderson et al. 2012). The HABs generated by phytoplankton (such as dinoflagellate) has extended the distribution range during the last decades and intensifying the frequency of this events (Anderson et al. 2021). Hence, there is an urgent need to develop methods with which to control algal expansion. In recent years, biological methods have become regarded as good approaches for regulating $\mathrm{HABs}$, largely because such methods may be more targeted and native to marine environments, affording better biosafety for aquatic organisms (Liao et al. 2014; Sengco and Anderson 2004). One such biological method is the use of algicidal bacteria; these have been shown to play an important role in lysing algal cells in aquatic ecosystems ( $\mathrm{Li}$ et al. 2015; Zhang et al. 2016). Most such bacteria have an algicidal effect that is relatively species-specific. In addition, algaecides that are derived from environmental

\footnotetext{
${ }^{\dagger}$ Corresponding authors: Y. Wang; wangyanmary@szpt.edu.cn and J. Zhou; zhou.jin@sz.tsinghua.edu.cn
}

The author(s) declare no conflict of interest.

Accepted for publication 2 March 2021.

\section{Funding}

This work was supported by the National Natural Science Foundation of China (41976126), Guangdong Basic and Applied Basic Research Foundation (2020B1515120012), and the S\&T Projects of Shenzhen Science and Technology Innovation Committee (JCYJ20200109142818589 and RCJC20200714114433069). 
microorganisms are very economical due to the low cost of the source material and because they are ecologically friendly (Zhou et al. 2010).

In this work, environmental samples were collected from the rhizosphere of mangrove Kandelia candel on the Shenzhen coast of China. Samples were serial diluted $\left(10^{-1}\right.$ to $\left.10^{-7}\right)$ in sterilized sea water, were spread onto 2216E agar plates (BD Diagnostic Systems), and were then incubated at $30^{\circ} \mathrm{C}$ for 48 to $72 \mathrm{~h}$. After incubation, colonies were randomly selected according to their morphology, size, and color. The bacteria colony was subcultured on sterile $2216 \mathrm{E}$ agar to obtain a pure culture of the bacterial isolate. After this, the bacterial strains were transferred to 1.5-ml EP tubes containing 2216E liquid medium and were then incubated for 24 to 48 h. Algicidal rate was measured according to a previously published method (Zhang et al. 2016). Briefly, it was calculated according to the following equation: algicidal rate $(\%)=\left(N_{C}-N_{E}\right) / N_{C} \times$ 10 , where $N_{C}$ and $N_{E}$ represent the number of algal cells in the control and experimental groups, respectively. Algal cells were counted after fixing them with Lugol's iodine reagent. The algicidal rate of samples from the algae Alexandrium tamarense inoculation system was determined every $6 \mathrm{~h}$. Strain $\mathrm{KC} 1$ had the highest rate and, thus, was analyzed further in subsequent experiments. Sequence analysis of the $16 \mathrm{~S}$ ribosomal RNA (rRNA) gene revealed that it shared $99.8 \%$ similarity to the type strain of Stenotrophomonas rhizophila. We provisionally named this strain S. rhizophila KC1. Currently, 13 assemblies of $S$. rhizophila at different levels are publicly available in GenBank, but only three of them have had their complete genomes sequenced. The three complete genomes were S. rhizophila GA1, S. rhizophila QL-P4, and S. rhizophila JC1. The main biological functions of them were inhibiting algal growth, resistance to heavy metals, and degradation of polyvinyl alcohol (Wei et al. 2017; Yin et al. 2018). Previously, several members of the genus Stenotrophomonas have been sequenced, many based on specimens collected from phycosphere environments (Karimi et al. 2020; Yin et al. 2018). To fully understand the molecular information of $S$. rhizophila strains, complete genome assemblies with fewer contigs and improved sequencing quality are essential. Additionally, genome sequencing of this strain will provide greater options for more effective identification of algicidal bacteria, the study of genes of interest (such as those involved in host recognition), and the development of specific molecular markers for population genetic diversity analysis.

Genomic DNA of S. rhizophila KC1 was extracted using a genomic DNA extraction kit (MoBio), following the protocols of the manufacturer. The quality of the DNA was checked on agarose gel while the quantity was measured using NanoDrop (Thermo Scientific NanoDrop lite spectrophotometer), which indicated $85 \mathrm{ng}$ per liter. Whole-genome sequencing of the normalized DNA was performed using the Illumina Hiseq 2500 instrument, as described by Glushchenko et al. (2017). The low-quality reads were filtered using Timmomatic version 1.2.14 (Bolger et al. 2014). De novo assembly was performed using CLC Genomics Workbench version 5.1 (CLC Bio) and was trimmed using a minimum Phred quality score of 20 , a minimum length of $50 \mathrm{bp}$, allowing no ambiguous nucleotides, and trimming off some lowquality nucleotides (Bankevich et al. 2012). The reads were assembled with SOAPdenovo (V.2.04) (Li et al. 2010), and sequences were annotated using the RAST annotation server (Aziz et al. 2008). Transfer RNA ( tRNA) and rRNA genes were predicted using tRNAscanSE (Lowe and Eddy 1997) and RNAmmer (Lagesen et al. 2007), respectively. Genes were predicted using Glimmer 3.02 (Delcher et al. 2007) and were annotated by searching against the National Center for Biotechnology Information (NCBI) nonredundant and Kyoto Encyclopedia of Genes and Genomes (KEGG) databases.

Figure 1 presents a circular map of $S$. rhizophila $\mathrm{KC1}$ and Table 1 lists the features of its whole genome. The final whole genome contained $5,234,745$ bases and a $\mathrm{G}+\mathrm{C}$ content of $63.17 \%$. There were 11,586 protein-coding gene sequences with an average size of 928 bp, giving a coding intensity of $88.28 \%$. Assembly of the genome yielded one contig. Further, 134 non-coding RNAs were found in the genome, including 73 tRNAs, 17 rRNAs, and 44 small RNAs (sRNAs). To study homology, we conducted BLAST searches of the NCBI database, which revealed 4,346 coding sequences (CDS) comprising 22 functional clusters of orthologous groups and included 39 KEGG metabolic pathways. Among the CDS, the five most abundant groups in the category of molecular function were amino acid metabolism (342 genes), carbohydrate metabolism (322 genes), translation, ribosomal structure and biogenesis (314 genes), signal transduction mechanisms (242 genes), and transport processes (226 genes), suggesting that two major groups of proteins in this bacterial species are those involved in cellular function and metabolism. 


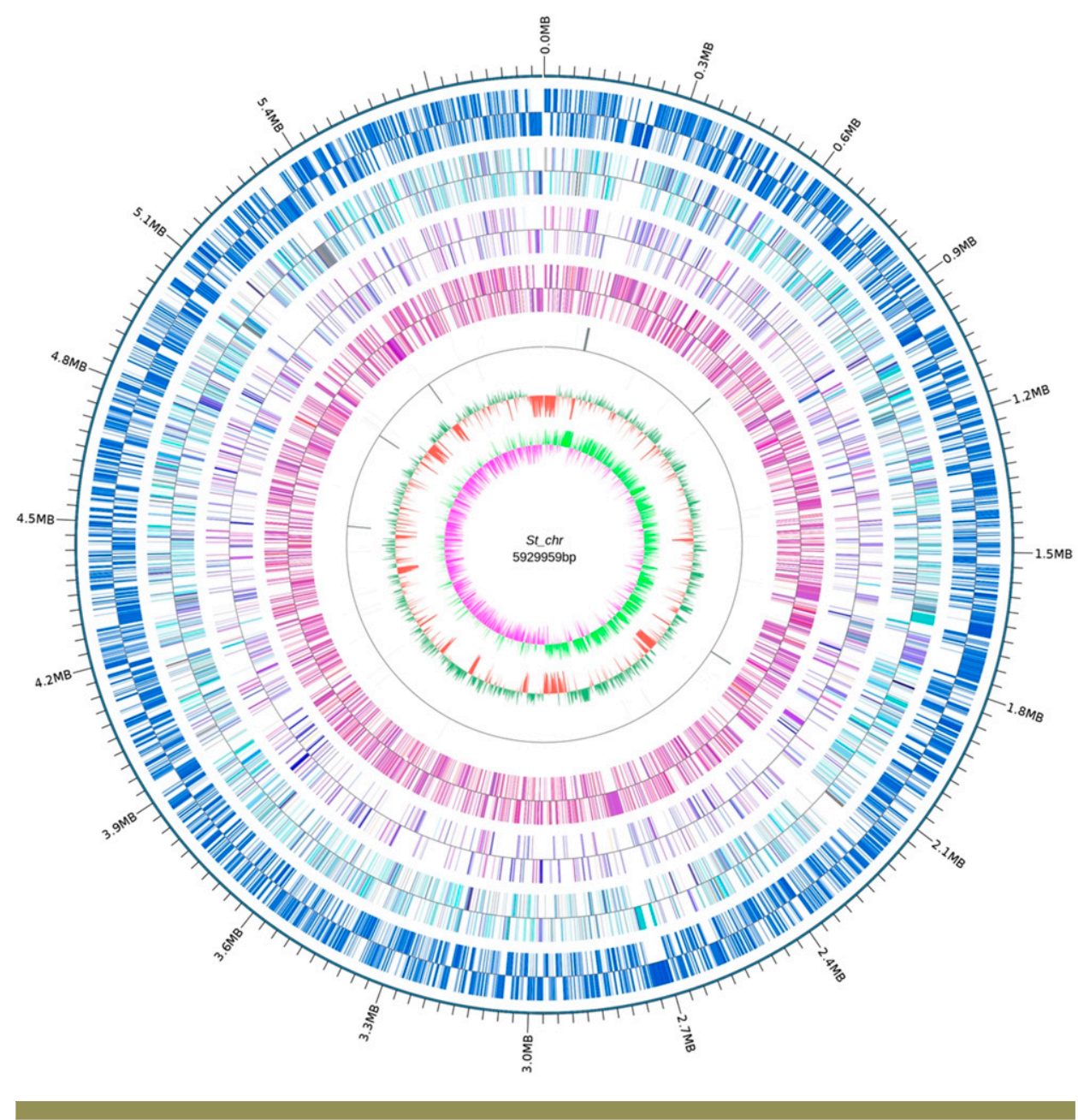

Fig. 1. Circular map for the whole genome of Stenotrophomonas rhizophila KC1. From the outside to the center: encoding genes, predicted coding sequences transcribed in the clockwise (or counter clockwise) direction, nondoding RNA, GC percent (\%), and GC skew (G+C/G-C) in a 1,000-bp window.

The quorum sensing (QS) signaling pathway usually mediates inter-species communication by producing small extracellular signal molecules (Even-Tov et al. 2016). Previously, Yin et al (2018) reported that algicidal bacterium S. rhizophila GA1 has multiple putative QS-like genes located in the upstream position at contig 4. Compared with GA1, there are 83 QS genes in the genome of $S$. rhizophila $\mathrm{KC1}$, which indicated $S$. rhizophila $\mathrm{KC1}$ has more potentiality to regulate its biological behavior under QS. The best-studied QS protein in gram-negative bacteria is $\mathrm{N}$-acyl homoserine lactone (AHL). To investigate AHL signaling, we performed in silico annotation to identify candidate Al-1 genes based on BLAST analyses. Two genes, encoding a pair of putative Luxl-LuxR genes, were subsequently found in the $S$. rhizophila $\mathrm{KC} 1$ isolate. Bioinformatics analyses confirmed the presence of essential AHL-related genes. The Luxl- and LuxR-encoding genes are 509 and 443 bp, respectively, suggesting their potential ability to manipulate cell density via AHL signaling (Martínez et al. 2015). In addition, LuxI is frequently detected in species that are closely related to algaeassociated bacteria (Zhang et al. 2020). Moreover, a cellulase-coding gene (cel) was found downstream of LuxI-LuxR; the protein encoded by this gene is believed to contribute to the ability of S. rhizophila KC1 to lyse its host. Accordingly, this microorganism is a potential algicidal bacterium with the ability to lyse algae.

In conclusion, the whole-genome sequence dataset generated in this study provides a deeper understanding of the relationships between bacteria and algae under the control of AHLs and may facilitate the development of new microbe-based methods for controlling HABs. The whole-genomics data can supply evidence to the ability of S. rhizophila KC1 in enhancing intraspecific communication and algicidal ability, thus making it a potential candidate to control for 
Table 1. General genome features of Stenotrophomonas rhizophila KC1 and MIGS mandatory information

\begin{tabular}{lc} 
Item & Description \\
Sequencing method & Illumina Heseq 2000 \\
Assembly & SOAPdenovo V.2.04 \\
Genome size & $5,929,959$ bp \\
GC content (\%) & 63.17 \\
Number of contigs & 1 \\
Total contig size & $5,234,745$ bp \\
Protein-coding genes & 11,586 \\
Transfer RNAs & 73 \\
Ribosomal RNAs & 17 \\
Small RNAs & 44 \\
Genes with a predicted function & 22 \\
Presumptive quorum-sensing genes & 83 \\
Potential Luxl-LuxR encoding site & Contig 1 \\
Encoding-Luxl and LuxR gene lengths & 509 and 443 bp \\
\hline
\end{tabular}

harmful algae. Although the specific substances responsible for this algicidal activity remain unidentified, our data can supply further evidence that $\mathrm{AHL}$ has the ability to regulate algicidal behavior. The signal-mediated algicidal system may have potential influence on algalbacterial interactions and aid in the design of strategies with which to control harmful algae blooms.

\section{Data Availability}

The S. rhizophila KC1 strain was deposited in the Marine Culture Collection of China with the accession number 1F08522. The complete genome sequence of S. rhizophila KC1 is available in GenBank under accession number CP065517.

\section{Author-Recommended Internet Resource}

GenBank S. rhizophila JC1 chromosome: https://www.ncbi.nlm.nih.gov/nuccore/NZ_CP050062

\section{Literature Cited}

Anderson, D. M. 2009. Approaches to monitoring, control and management of harmful algal blooms (HABs). Ocean Coast. Manage. 52:342-347.

Anderson, D. M., Cembella, A. D., and Hallegraeff, G. M. 2012. Progress in understanding harmful algal blooms: Paradigm shifts and new technologies for research, monitoring, and management. Annu. Rev. Mar. Sci. 4:143-176.

Anderson, D. M., Fensin, E., Gobler, C. J., Hoeglund, A. E., Hubbard, K. A., Kulis, D. M., Landsberg, J. H., Lefebvre, K. A., Provoost, P., Richlen, M. L., Smith, J. L., Solow, A. R., and Trainer, V. L. 2021. Marine harmful algal blooms (HABs) in the United States: History, current status and future trends. Harmful Algae 102:101975.

Aziz, R. K., Bartels, D., Best, A. A., DeJongh, M., Disz, T., Edwards, R. A., Formsma, K., Gerdes, S., Glass, E. M., Kubal, M., Meyer, F., Olsen, G. J., Olson, R., Osterman, A. L., Overbeek, R. A., McNeil, L. K., Paarmann, D., Paczian, T., Parrello, B., Pusch, G. D., Reich, C., Stevens, R., Vassieva, O., Vonstein, V., Wilke, A., and Zagnitko, O. 2008. The RAST server: Rapid annotations using subsystems technology. BMC Genomics 9:75.

Bankevich, A., Nurk, S., Antipov, D., Gurevich, A. A., Dvorkin, M., Kulikov, A. S., Lesin, V. M., Nikolenko, S. I., Pham, S., Prjibelski, A. D., Pyshkin, A. V., Sirotkin, A. V., Vyahhi, N., Tesler, G., Alekseyev, M. A., and Pevzner, P. A. 2012. SPAdes: A new genome assembly algorithm and its applications to single-cell sequencing. J. Comput. Biol. 19:455-477.

Bolger, A. M., Lohse, M., and Usadel, B. 2014. Trimmomatic: A flexible trimmer for Illumina sequence data. Bioinformatics 30:2114-2120.

Delcher, A. L., Bratke, K. A., Powers, E. C., and Salzberg, S. L. 2007. Identifying bacterial genes and endosymbiont DNA with Glimmer. Bioinformatics 23:673-679.
Even-Tov, E., Bendori, S. O., Valastyan, J., Ke, X., Pollak, S., Bareia, T., Ben-Zion, I., Bassler, B. L., and Eldar, A. 2016. Social evolution selects for redundancy in bacterial quorum sensing. PLoS Biol. 14:e1002386.

Glushchenko, O. E., Samoilov, A. E., Olekhnovich, E. I., Kovarsky, B. A., Tyakht, A. V., Pavlenko, A. V., Babenko, V. V., Larin, A. K., Kostryukova, E. S., Malakhova, M. V., llina, E. N., Abdulkhakov, R. A., Safina, D. I., Grigoryeva, T. V., Abdulkhakov, S. R., and Govorun, V. M. 2017. Data on gut metagenomes of the patients with Helicobacter pylori infection before and after the antibiotic therapy. Data Brief 11:68-71.

Karimi, E., Geslain, E., KleinJan, H., Tanguy, G., Legeay, E., Corre, E., and Dittami, S. M. 2020. Genome sequences of 72 bacterial strains isolated from Ectocarpus subulatus: A resource for algal microbiology. Genome Biol. Evol. 12:3647-3655.

Lagesen, K., Hallin, P., Rødland, E. A., Staerfeldt, H. H., Rognes, T., and Ussery, D. W. 2007. RNAmmer: Consistent and rapid annotation of ribosomal RNA genes. Nucleic Acids Res. 35:3100-3108.

Li, Y., Hu, Y., Bolund, L., and Wang, J. 2010. State of the art de novo assembly of human genomes from massively parallel sequencing data. Hum. Genomics 4:271.

Li, Z., Geng, M., and Yang, H. 2015. Algicidal activity of Bacillus sp. Lzh-5 and its algicidal compounds against Microcystis aeruginosa. Appl. Microbiol. Biotechnol. 99:981-990.

Liao, C., Liu, X., and Shan, L. 2014. Optimization of liquid media and biosafety assessment for algae-lysing bacterium NP23. Can. J. Microbiol. 60:593-597.

Lowe, T. M., and Eddy, S. R. 1997. tRNAscan-SE: A program for improved detection of transfer RNA genes in genomic sequence. Nucleic Acids Res. 25:955-964. 
Martínez, P., Huedo, P., Martinez-Servat, S., Planell, R., Ferrer-Navarro, M., Daura, X., Yero, D., and Gibert, I. 2015. Stenotrophomonas maltophilia responds to exogenous AHL signals through the LuxR solo SmoR (Smlt1839). Front. Cell. Infect. Microbiol. 5:41.

Medlin, L. 2013. Molecular tools for monitoring harmful algal blooms. Environ. Sci. Pollut. Res. Int. 20:6683-6685.

Sengco, M. R., and Anderson, D. M. 2004. Controlling harmful algal blooms through clay flocculation. J. Eukaryot. Microbiol. 51:169-172.

Wei, Y., Fu, J., Wu, J., Jia, X., Zhou, Y., Li, C., Dong, M., Wang, S., Zhang, J., and Chen, F. 2017. Bioinformatics Analysis and characterization of highly efficient polyvinyl alcohol (PVA)-degrading enzymes from the novel PVA degrader Stenotrophomonas rhizophila QL-P4. Appl. Environ. Microbiol. 84:e01898-17.
Yin, P., Zhang, Q., Zhu, J., Wu, G., Yin, S., Ma, Z., and Zhou, J. 2018. The data of genomic and phenotypic profiles of the $\mathrm{N}$-acyl homoserine lactoneproducing algicidal bacterium Stenotrophomonas rhizophila GA1. Data Brief 21:966-971.

Zhang, C., Li, Y., Meng, C. X., Yang, M. J., Wang, Y. G., Cai, Z. H., Zuo, P., and Zhou, J. 2020. Complete genome sequence of Acinetobacter baumanni J1, a quorum sensing-producing algicidal bacterium, isolated from eastern Pacific Ocean. Mar. Genomics 52:100719.

Zhang, H., Peng, Y., Zhang, S., Cai, G., Li, Y., Yang, X., Yang, K., Chen, Z., Zhang, J., Wang, H., Zheng, T., and Zheng, W. 2016. Algicidal effects of prodigiosin on the harmful algae Phaeocystis globosa. Front. Microbiol. 7:602.

Zhou, L., Chen, X., and Zheng, T. 2010. Study on the ecological safety of algacides: A comprehensive strategy for their screening. J. Appl. Psychol. 22:803-811. 